\title{
MicroRNA-146a-5p enhances cisplatin-induced apoptosis in ovarian cancer cells by targeting multiple anti-apoptotic genes
}

\author{
XIAODI LI ${ }^{1}$, YUHAO JIN ${ }^{1}$, ZEKUN MU $^{1}$, WEI CHEN ${ }^{2}$ and SONGSHAN JIANG ${ }^{1}$ \\ ${ }^{1}$ State Key Laboratory of Biocontrol, School of Life Sciences, Sun Yat-sen University; ${ }^{2}$ Department of Gynecology, \\ The Second Affiliated Hospital of Guangzhou Medical University, Guangzhou, Guangdong, P.R. China
}

Received March 1, 2017; Accepted April 26, 2017

DOI: 10.3892/ijo.2017.4023

\begin{abstract}
MicroRNAs play a crucial role in gene expression regulation in various types of cancers. Previous studies show the expression level of miR-146a-5p is downregulated in epithelial ovarian cancer. Further investigations suggest this downregulation is responsible for apoptosis resistance in ovarian cancer cells. However, the mechanism of how miR-146a-5p promotes apoptosis remains unclear. In this study, the role of miR-146a-5p in cisplatin-induced apoptosis of ovarian cancer cells was assessed by DAPI staining, MTT assays, and monitoring expression of XIAP, BCL2L2, BIRC2 and BIRC5 through a dual-luciferase assay. Our results show that miR-146a-5p can regulate three important anti-apoptotic genes including XIAP, BCL2L2 and BIRC5 via their 3'UTRs. Not only can overexpression of miR-146a-5p downregulate the expression of XIAP in SKOV3 cells, but it also lowers the $\mathrm{IC}_{50}$ values of cisplatin in OVCAR3 and SKOV3 cells and enhances the susceptibility of OVCAR3, SKOV3 and primary ovarian cancer cells to cisplatin-induced apoptosis. The effect of XIAP rescuing cisplatin-induced apoptosis accelerated by miR-146a-5p further supports our conclusion. Our results suggest that the regulation of three anti-apoptotic genes by miR-146-5p enhances the therapeutic effects of cisplatin.
\end{abstract}

\section{Introduction}

Epithelial ovarian cancer (EOC) is the third most common gynecological malignancy worldwide, and it is also one of the most fatal gynecological carcinomas in women (1), causing an estimated 125,000 deaths each year globally (2) and 14,240 deaths in the United States in 2015 alone (3). Currently,

Correspondence to: Dr Songshan Jiang, State Key Laboratory of Biocontrol, School of Life Sciences, Sun Yat-sen University, 132 East Waihuan Rd., Room 312, Higher Education Mega, Guangzhou, Guangdong 510006, P.R. China

E-mail: jiangssh@mail.sysu.edu.cn

Key words: microRNA-146a-5p, ovarian cancer, apoptosis, X-linked inhibitor of apoptosis protein, Baculoviral IAP repeat-containing protein 5, BCL2-like 2 platinum-based chemotherapy acts as the primary treatment for this cancer (4). Although patient prognosis has improved significantly for various types of solid cancers, women suffering from ovarian cancer exhibited only a slight change in survival rate since platinum-based treatment was introduced more than 30 years ago $(5,6)$. Furthermore, recent studies have revealed that some genetic and epigenetic alterations contribute to the survival of EOC (7). Thus, further understanding of the molecular basis of ovarian cancer is needed.

The anti-apoptotic effect resulting from the augmented expression of certain proteins involved in the apoptosis pathways is one of the most significant hallmarks for cancer cells. $\mathrm{X}$-linked inhibitor of apoptosis protein (XIAP), BCL2-like 2 (BCL2L2) and Baculoviral IAP repeat-containing protein 5 (BIRC5) are three important anti-apoptotic proteins in EOC development. Overexpression of XIAP, the most potent mammalian IAP, contributes to the abnormal survival of EOC cells via inhibiting caspase activity (8). Previous studies indicated that by downregulating XIAP, apoptosis is induced both in vitro and in vivo (9). Furthermore, XIAP has been found to be the target of miR-24 leading to the decrease of apoptotic threshold in cancer cells (10). BCL2L2, a pro-survival member of the BCL2 family of proteins, is associated with the ability of cancer cells to evade apoptotic signals $(11,12)$. Additionally, researchers have found that miR-15a can induce cell apoptosis by targeting BCL2L2 in non-small cell lung cancer (13), and miR-214 is able to enhance cisplatin-induced cytotoxicity via downregulation of BCL2L2 in cervical cancer cells (14).

Furthermore, a higher level of BCL2L2 also contributes to cancer cell resistance to drugs, such as camptothecin, cisplatin, etoposide (VP-16), adriamycin, or 1-D-arabinofuranosylcyto-sine (4). BIRC5 (survivin) is one of the eight well-studied members of the inhibitor of apoptosis protein (IAP) family, sharing a baculovirus IAP repeat (BIR), and has important roles in apoptosis (15). IAPs act as endogenous inhibitors of caspases, which are evolutionarily conserved (16,17). BIRC5 has a cell cycle-dependent expression pattern during mitosis, but it is also regulated by other non-cell cycle-dependent mechanisms (18). Previous studies suggested that BIRC5 counteracts apoptosis through interactions with caspases or initiators $(19,20)$. BIRC5 is strongly expressed in embryonic and fetal organs but not in most differentiated normal tissues $(21,22)$. Augmented expression 
of BIRC5 has been identified in tumors of breast, stomach, lung, pancreas and liver (23-27). Importantly, the aberrant expression of BIRC5 also correlates with human EOC in different aspects. BIRC5 promoter polymorphisms are found to be associated with age of onset of the disease (28). Furthermore, the overexpression of BIRC5 desensitizes EOC cell response to S-allylmercaptocysteine, a drug that inhibits the proliferation and metastasis of ovarian cancer cells (29). These findings make BIRC5 a key molecule in EOC research.

MicroRNA (miRNA), a type of small (20-25 nucleotides), noncoding RNA, acts as the main regulatory factor of gene expression by binding to the 3 '-untranslated regions (3'UTRs) of their targeted mRNAs (30), and this has been found to be relevant to various diseases in humans (31). To date, more than 2588 human miRNAs have been described in the miRBase database (http://www.mirbase.org/) with each miRNA possibly regulating the expression pattern of hundreds of target genes $(31,32)$. Moreover, numerous studies have shown that miRNA functions in oncogenic or cancer suppressor activities, including apoptosis (33). The downregulation of some miRNAs has been observed in a variety of cancers such as hepatocellular carcinoma and ovarian cancer, and others $(33,34)$. MicroRNA-146a-5p (miR-146a-5p) is a highly important miRNA, the dysregulation of which underlies the pathogenesis of peripartum cardiomyopathy, osteoarthritis, and the development or metastasis of various cancers, including papillary thyroid carcinoma and breast cancer (31,35-38). Additionally, miR-146a-5p is also critical for the suppressor function of Foxp $3^{+}$regulatory T cells, indicating the indispensability of a single miRNA in immune homeostasis (39). In ovarian cancer, Cui et al discovered that augmented expression of miR-146a-5p prohibits cell proliferation, enhances apoptosis, and increases sensitivity to chemotherapy drugs by reducing SOD2 (40).

Given the significant role of miR-146a-5p in EOC, we hypothesized that miR-146a-5p accelerates cisplatin-induced apoptosis via targeting certain anti-apoptotic genes. We found that miR-146a-5p lowers the $\mathrm{IC}_{50}$ value of cisplatin in OVCAR3 and SKOV3 ovarian cancer cell lines and promotes apoptosis in OVCAR3, SKOV3 and primary EOC cells. By computational predictions, two genes in the IAP family (XIAP and BIRC5) along with one gene in BCL2 family (BCL2L2) were predicted to be targets. Since miRNAs usually work by binding to their target mRNAs, a dual-luciferase assay was used to validate targets in our study. The results showed miR-146a-5p downregulates XIAP, BCL2L2 and BIRC5 via their 3'UTRs. After their 3'UTRs mutated, no differences were observed between the control group and miR-146a-5p-treated group, demonstrating that the $3^{\prime}$ UTRs are the regulatory site of miR-146a-5p. For the most strongly inhibited target XIAP, we further investigated the mechanism using a lentivirus packaging system, western blotting and DAPI staining. Overexpression of XIAP rescued the apoptosis-promoting effect of a miR-146a-5p mimic, and suppression of XIAP rescued the apoptosis-inhibiting effect of a miR-146a-5p inhibitor in a dose-dependent fashion. These data together suggest a pivotal role for miR-146a-5p in targeting several anti-apoptotic genes in ovarian cancer cells, and this suggests a mechanism that promotes apoptosis induced by cisplatin.

\section{Materials and methods}

Vector construction. To overexpress XIAP, human XIAP cDNA without its native 3'UTR was cloned downstream of the CMV promoter in the lentiviral expression vector pCDH-CMV-MCS-EF1-copGFP (pCDH; System Biosciences, Mountain View, CA, USA), and the construct was named LV-XIAP. To construct the luciferase reporter, the wild-type 3'UTRs of XIAP, BIRC2, BIRC5 and BCL2L2, containing the putative miR-146a-5p binding sites as well as the seed region mutated 3'UTR of XIAP, BIRC5 and BCL2L2, were fused to the Renilla luciferase reporter gene at the 3'UTR in the psiCHECK2 vector. The primers used for developing the constructs above are listed in Table I. DNA sequencing was performed to confirm all constructs.

Cell lines and cell culture. OVCAR3 and SKOV3 human ovarian cancer cell lines were purchased from the American Type Culture Collection (ATCC, Manassas, VA, USA) and were maintained in a $37^{\circ} \mathrm{C}, 5 \% \mathrm{CO}_{2}$ incubator in DMEM or RPMI (Invitrogen, Carlsbad, CA, USA) with $10 \%$ fetal bovine serum (FBS) and penicillin/streptomycin (Invitrogen) $(100 \mathrm{U} / \mathrm{ml})$. To isolate primary epithelial ovarian cancer cells from freshly collected malignant ascites from one patient, a previously described method was used (41). The pathological type of ovarian cancer of the patient was ovarian serous adenocystic carcinoma. The patient signed an informed consent form, and the use of the sample and study protocol were approved by the ethics committee of the Second Affiliated Hospital of Guangzhou Medical University (no. 2013034).

Transfection of RNA oligonucleotides. A miR-146a-5p mimic and its control RNA, a miR-146a-5p inhibitor and its control RNA, and an XIAP siRNA (si-XIAP) and its control RNA were synthesized, purified and annealed by GenePharma (Shanghai, China). The RNA sequences were as follows. miR-146a-5p mimic: sense 5'-UGAGAACUGAAUUCCAUGG GUU-3' and antisense 5'-CCCAUGGAAUUCAGUUCUC AUU-3'; mimic control: sense 5'-UUCUCCGAACGUGUC ACGUTT-3' and antisense 5'-ACGUGACACGUUCGGAG AATT-3'; miR-146a-5p inhibitor: 5'-AACCCAUGGAAUU CAGUUCUCA-3'; inhibitor control: 5'-CAGUACUUUUG UGUAGUACAA-3'; si-XIAP: sense 5'-CAUGCAGCUGUAG AUAGAUGGCAAU-3' and antisense 5'-AUUGCCAUCUAU CUACAGCUGCAUG-3'; siRNA control: sense 5'-UUCUC CGAACGUGUCACGUTT-3', antisense 5'-ACGUGACAC GUUCGGAGAATT-3'. RNA oligonucleotides (100 or $50 \mathrm{nM}$ ) were used with the X-tremeGENE siRNA Transfection Reagent (Roche, Basel, Switzerland) to transfect the miRNA mimic or siRNA into OVCAR3 and SKOV3 cells.

Western blotting. SKOV3 cells were seeded in 12-well plates at $2 \times 10^{5}$ cells/well and lysed using RIPA lysis buffer (BioTeke Corp., Beijing, China) $48 \mathrm{~h}$ post-transfection, and a bicinchoninic acid protein assay kit (Beyotime, Shanghai, China) was used to measure protein concentration. The protein sample (20 $\mu \mathrm{g}$ per lane) was first heat-denatured and then separated by $12 \%$ SDS-polyacrylamide gel electrophoresis. Protein was transferred to a PVDF membrane (Millipore, Bedford, MD, USA) and blocked with 3\% BSA. After blocking, the PVDF 
Table I. Primers used in this study.

\begin{tabular}{ll}
\hline Gene & \multicolumn{1}{c}{ Primer sequence (5' to 3') } \\
\hline LV-XIAP & S: CACAATCTAGAGCCACCATGACTTTTAACAGTTTTGAA \\
AS: AAGGATCCTTAAGACATAAAAATTTTTGCTTG & S: CACAACTCGAGCAGAGGAAAGTTTGAGAGTAAAACTG \\
XIAP-3'UTR2 & AS: AAGGATCCTATATCATGTGAAACTAATGCTGGGG \\
XIAP-mutant & S: TCCCAAGTCAAGAGAGTGTCTACATGTAGACTATTCCTTT \\
BCL2L2-3'UTR & AS: TAGACACTCTCTTGACTTGGGAGGGGGAAAAGATTTGGAT \\
BCL2L2-mutant & S: CACAACTCGAGGTGTGGGCACATGAAACGAC \\
AS: AAGGATCCATGCACAAGGAAGGGGGATG & S: GGGGGTCAAGAGTGTCCCTCCTCCCAACCC \\
BIRC2-3'UTR & AS: GACACTCTTGACCCCCTAGTTCTTGCCATT \\
BIRC5-3'UTR & S: CACAACTCGAGTGTTGAACACTTGAAGCCATCT \\
AS: AAGGATCCGCACCAAAGACAATTCGGCA & S: CACAACTCGAGTTGAAAGTGGCACCAGAGGT \\
BIRC5-mutant & AS: AAGGATCCCTTTCCACATGGCGACAGC \\
\hline & S: ACATGTGGTATTAAGAGCAAGAGTAAGTTGGAGTGGAGT \\
\hline
\end{tabular}

$\mathrm{S}$, Sense primer; AS, Antisense primer.

membrane was incubated for $2 \mathrm{~h}$ at room temperature with a mouse polyclonal antibody against human XIAP $(1: 10,000$; Santa Cruz Biotechnology, Santa Cruz, CA, USA) or a mouse monoclonal antibody against human $\beta$-actin (1:3000; Abcam, Cambridge, MA, USA) and incubated for $1 \mathrm{~h}$ with a goat anti-mouse (1:5000; Abcam) secondary antibody. To detect the bound antibodies, enhanced chemiluminescence detection reagents (Pierce, Rockford, IL, USA) were used. Band intensities were quantified with a Kodak Image Station 4000 MM Pro (Kodak, Tokyo, Japan).

Dual-luciferase assays. The dual-luciferase reporter assay was performed as previously described (42). First, 293T cells were seeded in a 96-well plate at $1 \times 10^{4}$ cells/well, and cells were co-transfected with $50 \mathrm{ng}$ of luciferase reporter vector and $6 \mathrm{ng}$ of synthetic miR-146a-5p mimic or mimic control. The cells were harvested $48 \mathrm{~h}$ post-transfection, and the luciferase activity was measured using a dual-luciferase reporter assay system (Promega, Madison, WI, USA) following the manufacturer's instructions. Renilla luciferase activities were normalized to firefly luciferase activities.

Assay of $I C_{50}$ value of cisplatin. The 3-(4,5)-dimethylthiazol(-2-y1)-3,5-di-phenyl-tetrazolium bromide (MTT) assay was used to measure the $\mathrm{IC}_{50}$ value of cisplatin. OVCAR3 and SKOV3 cells were transfected with miR-146a-5p mimic or mimic control one day after seeding into a 96-well plate at $1 \times 10^{4}$ cells/well. One day post-transfection, cisplatin was added to each well with a concentration gradient of $0.625,1.25$, $2.50,5.00,10.0,20.0$, and $40.0 \mu \mathrm{M}, 48 \mathrm{~h}$ later, $5 \mathrm{mg} / \mathrm{ml}$ of MTT reagent was added, and the plate was incubated in a $5 \%$
$\mathrm{CO}_{2}$ incubator at $37^{\circ} \mathrm{C}$ for $4 \mathrm{~h}$. DMSO $(100 \mu \mathrm{l})$ was added to each well, and the plate was mixed for $10 \mathrm{~min}$. The absorbance at $490 \mathrm{~nm}$ was detected with a BioTek microplate reader (Winooski, VT, USA).

4',6-Diamidino-2-phenylindole (DAPI) staining. OVCAR3, SKOV3 or primary EOC cells were seeded in a 48-well plate with a cell density of $1.5 \times 10^{4}$ cells per well and subsequently transfected with miR-146a-5p mimic or mimic control. To achieve $10-30 \%$ apoptosis, $5 \mu \mathrm{M}$ of cisplatin was added to the medium 16-24 h after transfection. After another 24-48 h, the cells were stained with $1 \mu \mathrm{g} / \mathrm{ml}$ DAPI (Sigma, St. Louis, MO, USA). A fluorescence microscope was used to observe apoptotic cells, and the apoptotic ratio was calculated from average 200 cellular nuclei in one image, and three images without overlap were taken randomly in each experiment. The average values \pm SD of three separate experiments were plotted.

Lentivirus packaging and infection. 293T cells were seeded in a $6-\mathrm{cm}$ dish at $8 \times 10^{5}$ cells/dish and co-transfected with $1.8 \mu \mathrm{g}$ of packaging plasmid pPAX2, $0.6 \mu \mathrm{g}$ of envelope plasmid pMD2.G and $2.5 \mu \mathrm{g}$ of the XIAP expression vector LV-XIAP or empty vector pCDH (LV-control) with the transfection reagent Lipofectamine 2000 (Invitrogen). Viral supernatants were harvested and stored at $-80^{\circ} \mathrm{C} 48 \mathrm{~h}$ post-transfection. Before infection, $0.01 \mu \mathrm{g} / \mathrm{ml}$ of polybrane (Sigma) was added in order to improve the infection efficiency.

Statistical analysis. The data were analyzed using Student's $\mathrm{t}$-test and are presented as the mean \pm standard deviation 
A

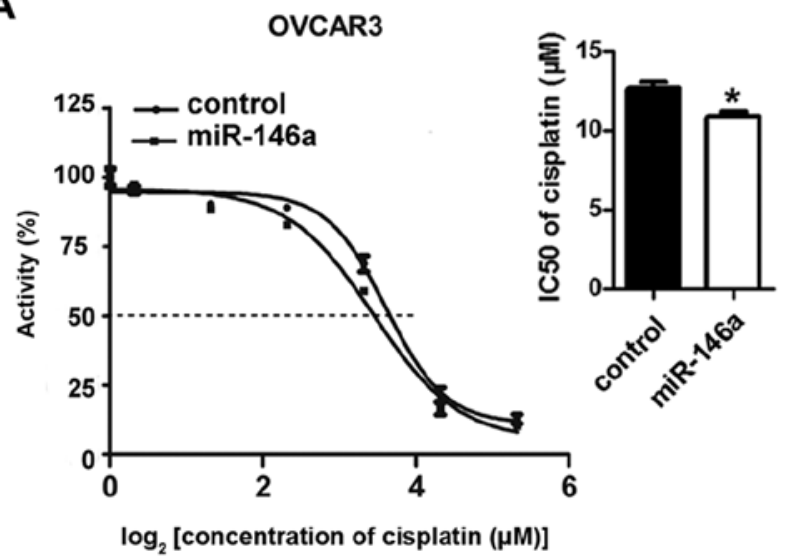

B

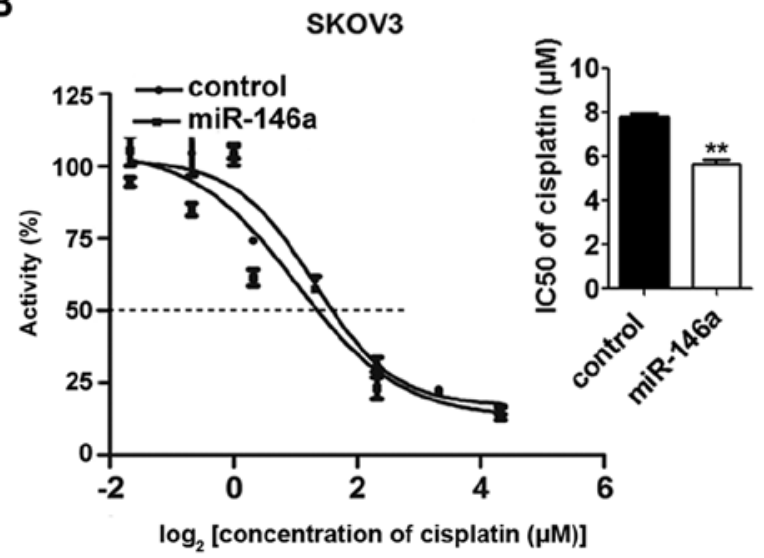

Figure 1. miR-146a-5p mimic can decrease the $\mathrm{IC}_{50}$ values of cisplatin in OVCAR3 and SKOV3 ovarian cancer cells. (A and B) Dose-response curves are presented. MTT assay was used to reflect the extent of apoptosis induced by cisplatin. Compared to control group, the $\mathrm{IC}_{50}$ value decreases when OVCAR3 cells (A) and SKOV3 cells (B) were transfected with miR-146a-5p mimic, respectively. The experiments were repeated 3 times. Significant differences from the control value are indicated by ${ }^{*} \mathrm{P}<0.05$ or ${ }^{* *} \mathrm{P}<0.01$.

$( \pm$ SD) of three separate experiments. P-values $<0.05,0.01$, or 0.001 indicate statistical significance. Prism software version 5.0 (GraphPad Software, La Jolla, CA, USA) was used to analyze the data.

\section{Results}

miR-146a-5p decreases the IC ${ }_{50}$ values of cisplatin in OVCAR3 and SKOV3 ovarian cancer cells. To investigate the function of miR-146a-5p in ovarian cancer cells, we determined the $\mathrm{IC}_{50}$ values of cisplatin in OVCAR3 and SKOV3 ovarian cancer cells transfected with miR-146a-5p mimic or mimic control. After transfection, cisplatin was added to each well with a concentration gradient of 40, 20, 10, 5, 2.5, 1.25, 0.625 and $0 \mu \mathrm{M}$ to evaluate the $\mathrm{IC}_{50}$ values. We found that for the two cell lines, the $\mathrm{IC}_{50}$ values decreases with transfection of miR-146a-5p, and this is most significant in the SKOV3 cell line (Fig. 1A and B). These results reveal that miR-146a-5p can effectively increase the sensitivity of OVCAR3 and SKOV3 ovarian cancer cells to cisplatin-induced apoptosis.

miR-146a-5p promotes apoptosis in OVCAR3, SKOV3 and primary ovarian cancer cells. We hypothesized that miR-146a-5p lowers $\mathrm{IC}_{50}$ values of cisplatin in ovarian cancer cells by promoting apoptosis. To test this effect, two ovarian cancer cell lines (OVCAR3 and SKOV3) and primary EOC cells were used. After seeding onto a 48-well plate, OVCAR3 cells were transfected with miR-146a-5p mimic, mimic control, miR-146a-5p inhibitor or inhibitor control. Cisplatin treatment and DAPI staining were performed as previously described (43). Our results show that more apoptotic nuclei were observed in OVCAR3 cells transfected with miR-146a-5p mimic compared to mimic control (Fig. 2A). Apoptosis was markedly suppressed in OVCAR3 cells transfected with miR-146a-5p inhibitor compared to the inhibitor control (Fig. 2B). These results indicate that miR-146a-5p is the driving force of the observed higher apoptosis rate.

In order to define the function of miR-146a-5p, SKOV3 cells were transfected with mimic control and miR-146a-5p mimic and treated with cisplatin. DAPI staining revealed a higher apoptosis ratio in SKOV3 cells transfected with
Table II. Predicted miR-146a-5p binding sites in the 3'UTR of anti-apoptotic genes.

\begin{tabular}{lll}
\hline Genes & $\begin{array}{c}\text { With miR-146a-5p } \\
\text { binding sites } \\
\text { in 3'UTR }\end{array}$ & $\begin{array}{c}\text { Without miR-146a-5p } \\
\text { binding sites } \\
\text { in 3'UTR }\end{array}$ \\
\hline IAP family & XIAP, BIRC2, & BIRC3, NAIP, BIRC6, \\
& BIRC5 & BIRC7, BIRC8 \\
BCL2 family & BCL2L2 & BCL2L1, BCL2, \\
& & CCND1, MCL1 \\
\hline
\end{tabular}

miR-146a-5p mimic compared to mimic control (Fig. 2C). Primary EOC cells isolated from ascites were also transfected with miR-146a-5p mimic or mimic control and treated with $5 \mu \mathrm{M}$ cisplatin for 24 or $48 \mathrm{~h}$. We found that the apoptosis rate in transfected primary cultured EOC cells was increased approximately $50 \%$ after $24 \mathrm{~h}$, and this rate almost doubled after another $24 \mathrm{~h}$ (Fig. 3). Together, these robust results strongly suggest that miR-146a-5p accelerates apoptosis by sensitizing EOC cells to cisplatin.

Screening of anti-apoptotic genes targeted by miR-146a-5p. Next, we wanted to identify genes targeted by miR-146a-5p. Given its role in accelerating apoptosis, we predicted that the target genes would be anti-apoptotic. Scores of studies have confirmed the tight connection between proteins of the IAP family and BCL2 family with carcinogenesis, including studies of ovarian cancer $(9,15,44-46)$. We hypothesized that miR-146a-5p could possibly regulate these two families, by decreasing expression of these proteins which are often overexpressed in chemoresistant EOC cells. To elucidate which proteins could be targeted by miR-146a-5p, a computational method was utilized to predict potential miR-146a-5p-binding anti-apoptotic genes (Table II). Four genes (XIAP, BIRC2 and BIRC5 from the IAP family and BCL2L2 from the BCL2 family) out of 13 candidate genes were predicted to have 
A
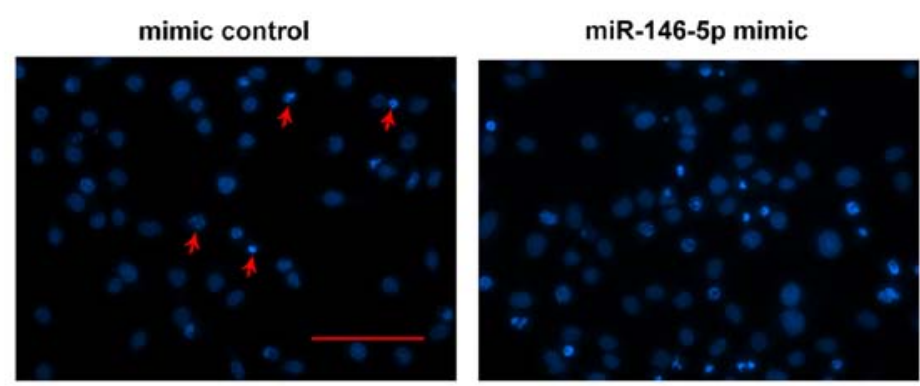

B
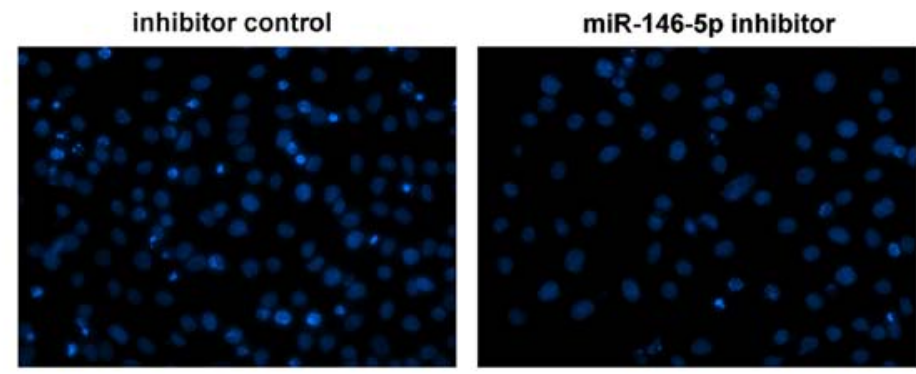

C

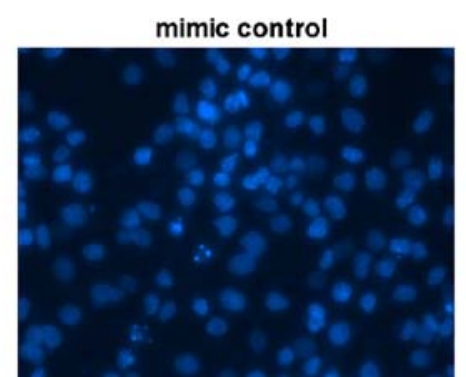

miR-146a-5p mimic

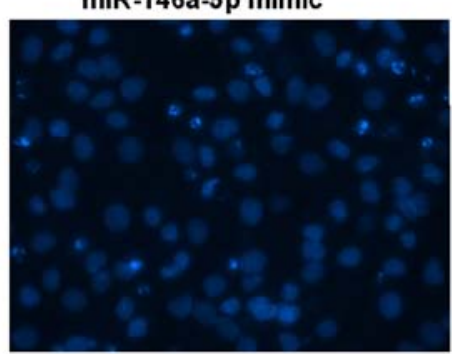

OVCAR3

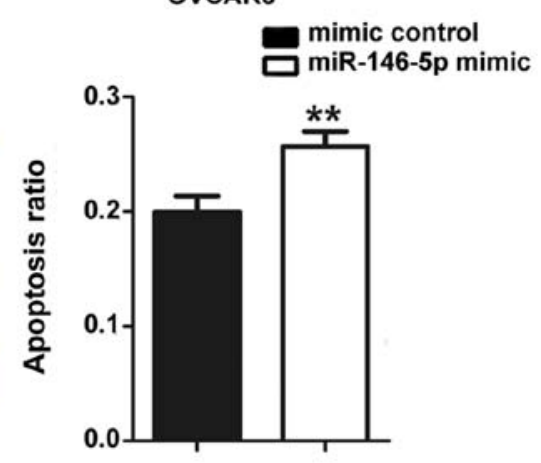

OVCAR3

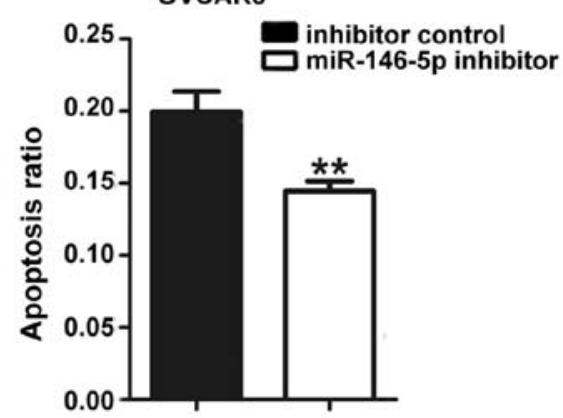

SKov3

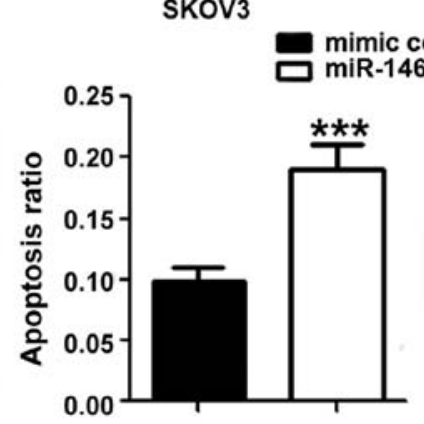

Figure 2. miR-146a-5p promotes apoptosis of OVCAR3 and SKOV3 cells. (A and B) OVCAR3 cells were transfected with miR-146a-5p mimic or mimic control (A) and miR-146a-5p inhibitor or inhibitor control (B), respectively. (C) SKOV3 cells were transfected with miR-146a-5p mimic or mimic control. The cells were stained with DAPI $48 \mathrm{~h}$ after cisplatin treatment, and apoptosis ratio was measured. Representative images are shown in the left panel (Scale bar, $100 \mu \mathrm{m}$ ), and the corresponding statistical results from three independent transfection experiments are shown in the right panel. The apoptotic cell nuclei were indicated with arrows. The average values \pm SD from three separate experiments were plotted, and significant differences from the control value are indicated by ${ }^{* *} \mathrm{P}<0.01$ or ${ }^{* * *} \mathrm{P}<0.001$.

miR-146a-5p binding sites in their 3'UTRs (Fig. 4A). XIAP and BIRC2 had one predicted site, while BCL2L2 and BIRC5 had two potential target sites.

A dual-luciferase reporter assay was used to test if miR-146a-5p regulates XIAP, BCLCL2, BIRC2 and BIRC5 by binding to their 3'UTRs. 293T cells were seeded in a 96-well plate $24 \mathrm{~h}$ before transfection, and cells were co-transfected with miR-146a-5p mimic or mimic control and the psiCHECK2 vector containing a luciferase reporter gene fused with the 3'UTR of each the four genes. After $48 \mathrm{~h}$ cells were harvested, and the luciferase activity was measured. The luciferase activity data showed that the miR-146a-5p mimic inhibited the three 3'UTRs of XIAP, BCL2L2 and BIRC5 to different extents (Student's t-test, $\mathrm{p}<0.05$ ) (Fig. 4B). This effect was most significant with XIAP. The results for BCL2L2 showed a moderate influence, which was followed by BIRC5. There was no interaction between miR-146a-5p and the 3'UTR of BIRC2.

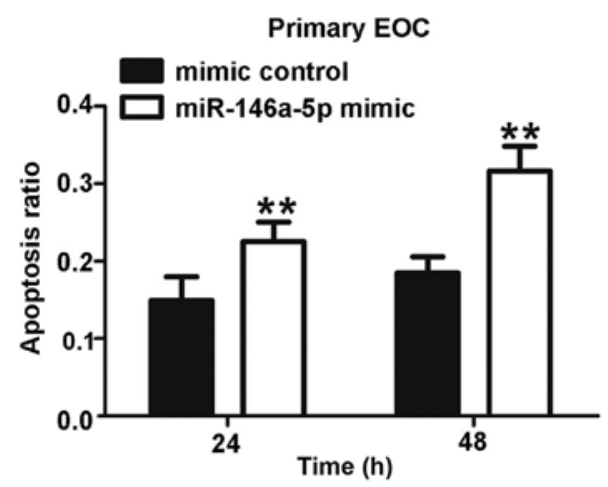

Figure 3. miR-146a-5p promotes apoptosis of primary EOC cells. Primary EOC cells were transfected with miR-146a-5p mimic or mimic control. The statistical results of DAPI staining at 24 or $48 \mathrm{~h}$ after cisplatin treatment are shown. The average values \pm SD from three separate experiments were plotted, and significant differences from the control group value are indicated by ${ }^{* *} \mathrm{P}<0.01$. 
A

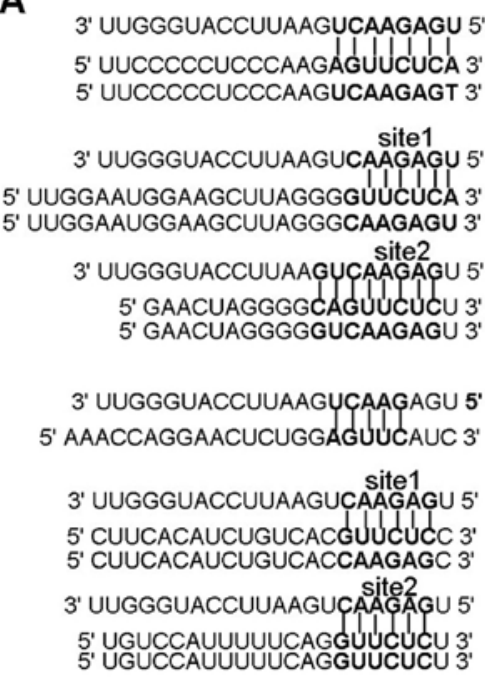

B

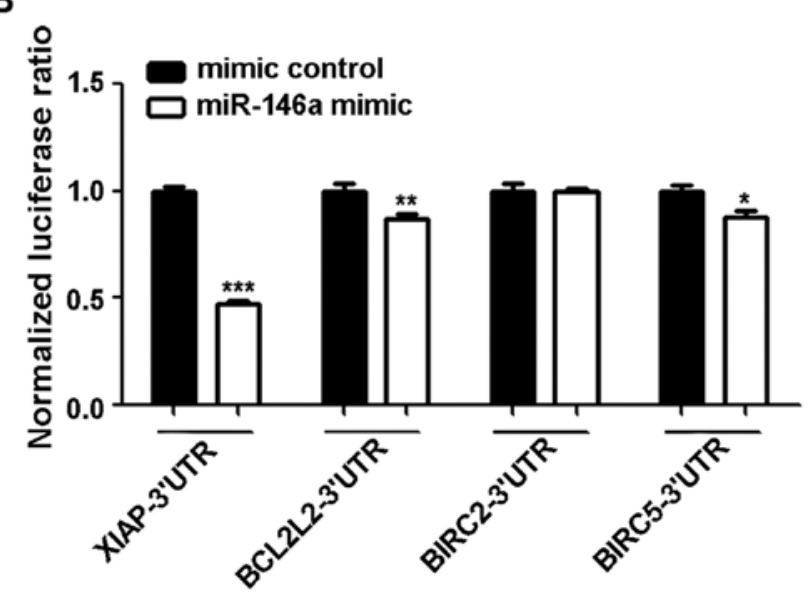

Figure 4. Screening of anti-apoptotic genes targeted by miR-146a-5p. (A) The targeting sites of miR-146a-5p on multiple anti-apoptotic genes were predicted, and the alignments of miR-146a-5p with their mRNA 3'UTRs are shown. (B) Dual-luciferase assay was utilized to test binding between miR-146a-5p and various anti-apoptotic genes. The values represent the mean \pm SD from three independent transfection experiments. Significant differences from the control value are designated by ${ }^{*} \mathrm{P}<0.05,{ }^{* *} \mathrm{P}<0.01$ or ${ }^{* * * *} \mathrm{P}<0.001$.

miR-146a-5p targets XIAP, BCL2L2 and BIRC5. To determine whether miR-146a-5p recognizes the predicted sites (XIAP 3'UTR 2781-2803; BCL2L2 3'UTR 394-420, 567-586; BIRC5 3'UTR 918-940, 1831-1852), we constructed the seed region-mutated Renilla luciferase reporter for XIAP 3'UTR, both seed region-mutated reporters for BCL2L2 and BIRC5 3'UTRs (Fig. 4A). For each gene, both wild-type and mutant reporters were co-transfected into $293 \mathrm{~T}$ cells with miR-146a-5p mimic or mimic control, respectively (Fig. 5A). By testing the luciferase activity, we found that the ability of miR-146a-5p to inhibit XIAP, BCL2L2 and BIRC5 were abrogated through a mutation for XIAP, and mutations of both sites for BCL2L2 and BIRC5 (Fig. 5A). Since XIAP is the most significantly inhibited gene, we chose XIAP for further experiments.

To confirm whether miR-146a-5p could also influence endogenous target expression, SKOV3 cells were transfected with miR-146a-5p mimic or mimic control. Western blot analysis indicated that the level of endogenous XIAP decreased, and this was ascribed to the transfection of miR-146a-5p mimic compared to the control group. The level of the internal reference GADPH was consistent between the two groups (Fig. 5B). Taken together, our data demonstrated that miR-146a-5p targets XIAP, BCL2L2 and BIRC5 via their 3'UTRs in 293T and ovarian cancer cells.

$X I A P$ rescues the effects of miR-146a-5p on apoptosis. We showed that miR-146a-5p promoted cisplatin-induced apoptosis in ovarian cancer cells (Fig. 2), and it was also able to inhibit the expression of XIAP. To further validate that miR-146a-5p indeed accelerates cisplatin-induced apoptosis via down-regulating XIAP, a rescue experiment was performed. OVCAR3 cells were co-transfected with $50 \mathrm{nM}$ si-XIAP and different amounts of miR-146a-5p inhibitor (100 and $200 \mathrm{nM}$ ). DAPI staining was used to allow quantification of the state of apoptosis. When $50 \mathrm{nM}$ si-XIAP and $100 \mathrm{nM}$ miR-146a-5p inhibitor was co-transfected, there was no significant difference between the $100 \mathrm{nM}$ miR-146a-5p inhibitor experimental group and control group, but there was weaker apoptotic induction in the $200 \mathrm{nM}$ miR-146a-5p inhibitor experimental group compared to the control group (Fig. 6A). This suggests that XIAP siRNA can counteract the effect of miR-146a-5p inhibitor in suppressing apoptosis, and there is decreased apoptosis when a higher amount of miR-146a-5p inhibitor is transfected. Similarly (Fig. 6B), OVCAR3 cells were infected with constant quantities of XIAP without its 3'UTR (multiplicities of infection equal to 1 ) and were then transfected with varying amounts of miR-146a-5p mimic (50 and $100 \mathrm{nM})$, and there was no obvious difference between $50 \mathrm{nM}$ miR-146a-5p mimic group and control group and a higher apoptosis ratio in $100 \mathrm{nM}$ miR-146a-5p mimic group compared to control group (Fig. 6B). These results indicate that XIAP can counteract the effect of miR-146a-5p in promoting apoptosis, and there is enhanced apoptosis when miR-146a-5p is expressed excessively. The western blot results from our previous study indicated that XIAP was overexpressed with lentiviral infection, and si-XIAP decreased XIAP expression in OVCAR3 cells (47). In summary, our results indicate that these four anti-apoptotic proteins are targets of miR-146a-5p, providing insights into how miR-146a-5p can promote apoptosis in EOC cells.

\section{Discussion}

Many studies have illustrated that miR-146a-5p is engaged in multiple diseases and the development of metastasis in various cancer types (31,35-38). Furthermore, augmented expression of miR-146a-5p can inhibit cell proliferation, enhance apoptosis, and increase chemosensitivity (40), which prompted us to speculate that miR-146a-5p may play a suppressive role in ovarian cancer. In support of this hypothesis, our results showed that overexpression of miR-146a-5p reduces the $\mathrm{IC}_{50}$ values of cisplatin in OVCAR3 and SKOV3 cells and enhances cisplatin-induced apoptosis in OVCAR3, SKOV3 and primary ovarian cancer cells.

XIAP, BCL2L2 and BIRC5 are three important anti-apoptotic proteins in a multitude of cancers $(8,38)$. Numerous studies have reported that XIAP, whose expression is at a high level in ovarian cancer (48-50), is essential for suppressing 
A

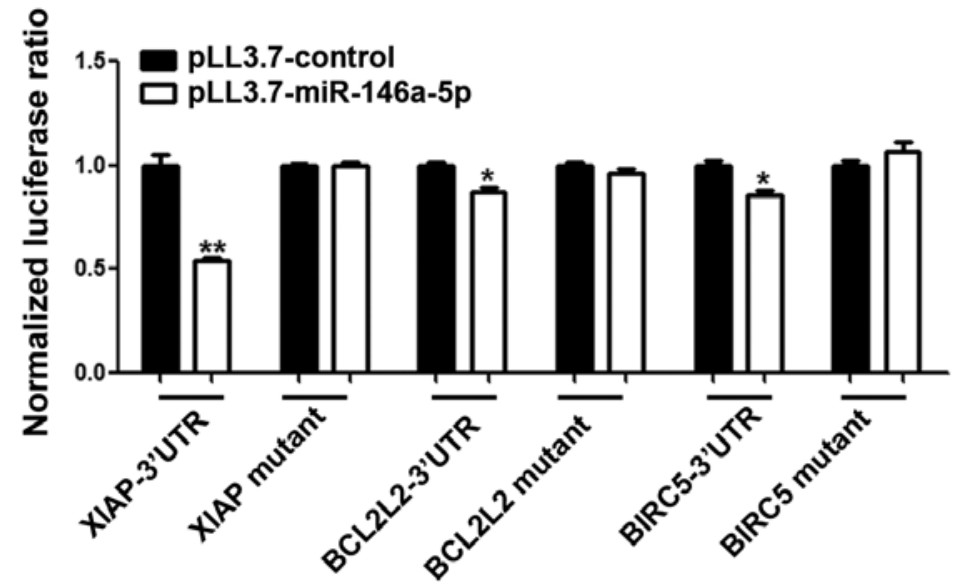

B

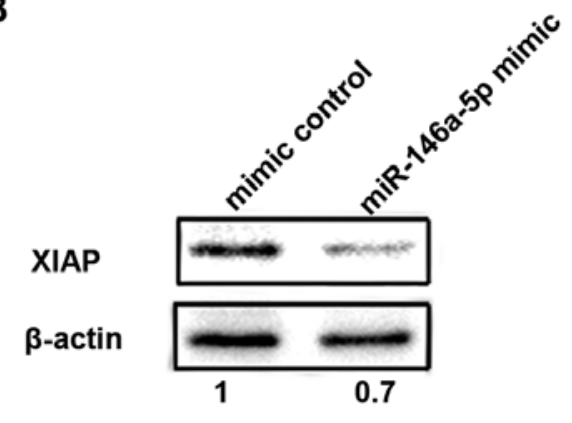

Figure 5. Authentication using potential mutation site. (A) Dual-luciferase assay was used to verify miR-146a-5p inhibition of the 3'UTRs of XIAP, BCL2L2 and BIRC5 with the co-transfection of seed region mutated 3'UTRs and miR-146a-5p mimic, respectively. (B) miR-146a-5p mimic was able to inhibit endogenous XIAP in SKOV3 ovarian cancer cells, which was detected by western blotting. ${ }^{*} \mathrm{P}<0.05,{ }^{* * *} \mathrm{P}<0.01$.

A

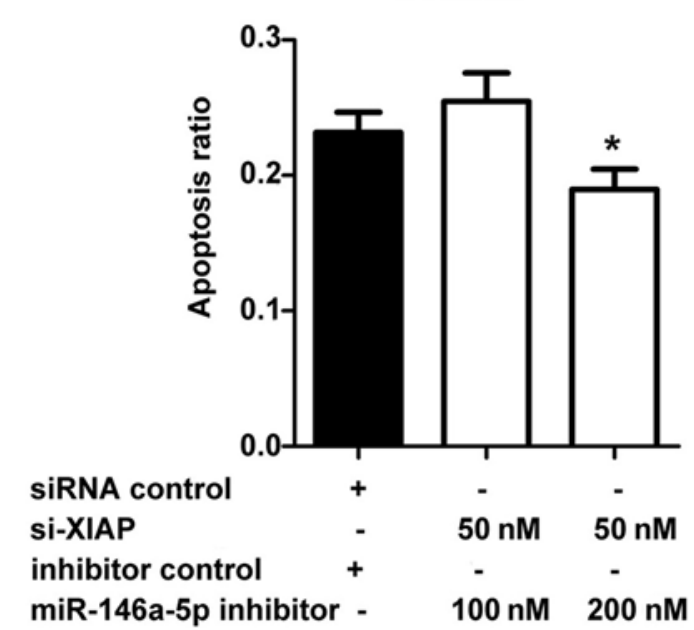

B

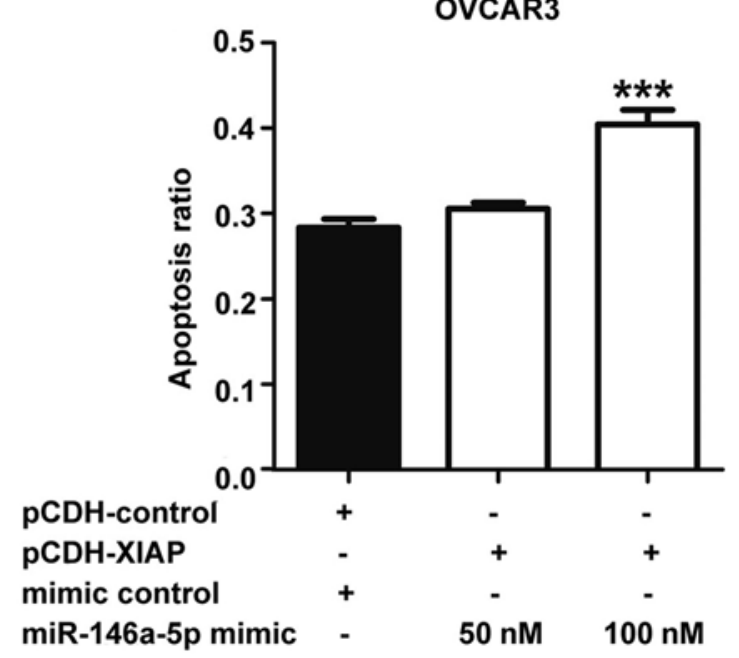

Figure 6. XIAP rescues apoptosis-promoting effect of miR-146a-5p. (A) OVCAR3 cells were co-transfected with different amounts of miR-146a-5p inhibitor and $50 \mathrm{nM}$ of si-XIAP. We later treated the cells with cisplatin as previously described. DAPI staining was used to indicate nuclei $48 \mathrm{~h}$ after transfection. The statistical analysis is shown. (B) OVCAR3 cells were infected with lentivirus expressing XIAP with multiplicities of infection equal to 1 and then transfected with different amounts of miR-146a-5p mimic. Cisplatin was used to induce apoptosis. The statistical analysis of $\mathrm{DAPI}$ staining is shown. $\mathrm{P}<0.05$, $^{* *} \mathrm{P}<0.01$.

apoptosis and promoting cell proliferation (8). Likewise, BIRC5 also have functions associated with apoptosis $(19,51)$. Overexpression of BIRC5 can decrease the sensitivity of EOC cells to an anti-proliferative drug (29). BCL2L2 is also associated with overriding apoptotic signals in cancer cells $(11,12)$. Bioinformatic predictions and luciferase assays demonstrated that these three proteins can be targeted by miR-146a-5p via their 3'UTRs, and the effect on XIAP was the most significant (Figs. 4 and 5). Furthermore, the experiments showed that XIAP functionally rescued the apoptotic effect of miR-146a-5p (Fig. 6). Consequently, we deduced that the potentially abnormal functions of these three proteins in ovarian cancer might be partially ascribed to the dysregulation of some miRNAs including miR-146a-5p. Several studies have illustrated that XIAP is also regulated by other miRNAs such as miR-519d, miR-509-3p, miR-155 and miR-7 (43,52-54), which may allow us to construct a XIAP-regulating network.
Furthermore, this study provided novel targets for EOC treatment. The five-year relative survival rate of ovarian cancer patients is $46 \%$ from 2005 to 2011, without much increase compared to that from 1987 to 1989 at 36\% (3). Currently, standard platinum chemotherapy leads to a recurring drug-resistant cancer in approximately $25 \%$ of patients within six months (55). This relatively unsatisfactory prognosis means that further understanding of the molecular mechanisms of ovarian cancer is needed. Previous research revealed that overexpression of miR-146a-5p in EOC cells inhibits cell proliferation and enhances apoptosis and chemosensitivity through reduction of SOD2 (40). Herein, data from our work further showed that miR-146a-5p decreases the $\mathrm{IC}_{50}$ value of cisplatin in ovarian cancer cells by downregulating three anti-apoptotic genes including XIAP. Thus, augmenting the level of miR-146a-5p by gene therapy combined with cisplatin chemotherapy might be a more efficient treatment. 
In conclusion, our study indicates that miR-146a-5p promotes cisplatin-induced apoptosis in ovarian cancer cells by repressing multiple anti-apoptotic genes, including XIAP, BCL2L2 and BIRC5, of which XIAP shows the strongest evidence. The miR-146a-5p/three protein axis should be further tested by evaluation of whether miR-146a-5p level correlates with prognosis using clinical specimens. These studies may illuminate new targets for treatment of ovarian cancer, which may facilitate cisplatin-induced apoptosis and improve chemotherapy.

\section{Acknowledgements}

This study was supported by grants from the Natural Science Foundation of China (grant no. 81572567).

\section{References}

1. Jemal A, Bray F, Center MM, Ferlay J, Ward E and Forman D: Global cancer statistics. CA Cancer J Clin 61: 69-90, 2011.

2. Cannistra SA: Cancer of the ovary. N Engl J Med 351: 2519-2529, 2004.

3. Siegel RL, Miller KD and Jemal A: Cancer statistics, 2016. CA Cancer J Clin 66: 7-30, 2016.

4. Zhou Y, Chen Q, Qin R, Zhang K and Li H: MicroRNA-449a reduces cell survival and enhances cisplatin-induced cytotoxicity via downregulation of NOTCH1 in ovarian cancer cells. Tumour Biol 35: 12369-12378, 2014.

5. Vaughan S, Coward JI, Bast RC Jr, Berchuck A, Berek JS, Brenton JD, Coukos G,Crum CC,Drapkin R,Etemadmoghadam D, et al: Rethinking ovarian cancer: Recommendations for improving outcomes. Nat Rev Cancer 11: 719-725, 2011.

6. McGuire WP: Maintenance therapy for ovarian cancer: Of Helsinki and Hippocrates. J Clin Oncol 27: 4633-4634, 2009.

7. Kurman RJ, Visvanathan K, Roden R, Wu TC and Shih IeM Early detection and treatment of ovarian cancer: Shifting from early stage to minimal volume of disease based on a new model of carcinogenesis. Am J Obstet Gynecol 198: 351-356, 2008.

8. Kiraz Y, Adan A, Kartal Yandim M and Baran Y: Major apoptotic mechanisms and genes involved in apoptosis. Tumour Biol 37: 8471-8486, 2016.

9. Shaw TJ, Lacasse EC, Durkin JP and Vanderhyden BC: Downregulation of XIAP expression in ovarian cancer cells induces cell death in vitro and in vivo. Int J Cancer 122: 1430-1434, 2008.

10. Xie Y, Tobin LA, Camps J, Wangsa D, Yang J, Rao M, Witasp E, Awad KS, Yoo N, Ried T, et al: MicroRNA-24 regulates XIAP to reduce the apoptosis threshold in cancer cells. Oncogene 32: 2442-2451, 2013.

11. Frenzel A, Grespi F, Chmelewskij W and Villunger A: Bcl2 family proteins in carcinogenesis and the treatment of cancer. Apoptosis 14: 584-596, 2009.

12. Camisasca DR, Honorato J, Bernardo V, da Silva LE, da Fonseca EC, de Faria PA, Dias FL and Lourenço SQ: Expression of Bcl-2 family proteins and associated clinicopathologic factors predict survival outcome in patients with oral squamous cell carcinoma. Oral Oncol 45: 225-233, 2009.

13. Yang T, Thakur A, Chen T, Yang L, Lei G, Liang Y, Zhang S, Ren $\mathrm{H}$ and Chen M: MicroRNA-15a induces cell apoptosis and inhibits metastasis by targeting BCL2L2 in non-small cell lung cancer. Tumour Biol 36: 4357-4365, 2015.

14. Wang F, Liu M, Li X and Tang H: MiR-214 reduces cell survival and enhances cisplatin-induced cytotoxicity via down-regulation of Bcl212 in cervical cancer cells. FEBS Lett 587: 488-495, 2013.

15. Cory S, Huang DCS and Adams JM: The Bcl-2 family: Roles in cell survival and oncogenesis. Oncogene 22: 8590-8607, 2003.

16. Salvesen GS and Duckett CS: IAP proteins: Blocking the road to death's door. Nat Rev Mol Cell Biol 3: 401-410, 2002.

17. Saleem M, Qadir MI, Perveen N, Ahmad B, Saleem U, Irshad T and Ahmad B: Inhibitors of apoptotic proteins: New targets for anticancer therapy. Chem Biol Drug Des 82: 243-251, 2013.

18. Altieri DC: Validating survivin as a cancer therapeutic target. Nat Rev Cancer 3: 46-54, 2003.
19. Tamm I, Wang Y, Sausville E, Scudiero DA, Vigna N, Oltersdorf T and Reed JC: IAP-family protein survivin inhibits caspase activity and apoptosis induced by Fas (CD95), Bax, caspases, and anticancer drugs. Cancer Res 58: 5315-5320, 1998

20. Kasof GM and Gomes BC: Livin, a novel inhibitor of apoptosis protein family member. J Biol Chem 276: 3238-3246, 2001.

21. Adida C, Crotty PL, McGrath J, Berrebi D, Diebold J and Altieri DC: Developmentally regulated expression of the novel cancer anti-apoptosis gene survivin in human and mouse differentiation. Am J Pathol 152: 43-49, 1998.

22. Ambrosini G, Adida C and Altieri DC: A novel anti-apoptosis gene, survivin, expressed in cancer and lymphoma. Nat Med 3: 917-921, 1997.

23. Monzó M, Rosell R, Felip E, Astudillo J, Sánchez JJ, Maestre J, Martín C, Font A, Barnadas A and Abad A: A novel anti-apoptosis gene: Re-expression of survivin messenger RNA as a prognosis marker in non-small-cell lung cancers. J Clin Oncol 17: 2100-2104, 1999.

24. Tanaka K, Iwamoto S, Gon G, Nohara T, Iwamoto M and Tanigawa N: Expression of survivin and its relationship to loss of apoptosis in breast carcinomas. Clin Cancer Res 6: 127-134, 2000.

25. Lu CD, Altieri DC and Tanigawa N: Expression of a novel antiapoptosis gene, survivin, correlated with tumor cell apoptosis and p53 accumulation in gastric carcinomas. Cancer Res 58: 1808-1812, 1998.

26. Satoh K, Kaneko K, Hirota M, Masamune A, Satoh A and Shimosegawa T: Expression of survivin is correlated with cancer cell apoptosis and is involved in the development of human pancreatic duct cell tumors. Cancer 92: 271-278, 2001.

27. Ikeguchi M, Ueta T, Yamane Y, Hirooka Y and Kaibara N: Inducible nitric oxide synthase and survivin messenger RNA expression in hepatocellular carcinoma. Clin Cancer Res 8: 3131-3136, 2002

28. Han CH, Wei Q, Lu KK, Liu Z, Mills GB and Wang LE: Polymorphisms in the survivin promoter are associated with age of onset of ovarian cancer. Int J Clin Exp Med 2: 289-299, 2009.

29. Wu J, Zhao S, Zhang J, Qu X, Jiang S, Zhong Z, Zhang F, Wong $Y$ and Chen $\mathrm{H}$ : Over-expression of survivin is a factor responsible for differential responses of ovarian cancer cells to S-allylmercaptocysteine (SAMC). Exp Mol Pathol 100: 294-302, 2016.

30. Bartel DP: MicroRNAs: Genomics, biogenesis, mechanism, and function. Cell 116: 281-297, 2004.

31. Rusca N and Monticelli S: MiR-146a in immunity and disease. Mol Biol Int 2011: 437301, 2011.

32. Navarro F and Lieberman J: Small RNAs guide hematopoietic cell differentiation and function. J Immunol 184: 5939-5947, 2010.

33. Nam EJ, Yoon H, Kim SW, Kim H, Kim YT, Kim JH, Kim JW and Kim S: MicroRNA expression profiles in serous ovarian carcinoma. Clin Cancer Res 14: 2690-2695, 2008.

34. Hou J, Lin L, Zhou W, Wang Z, Ding G, Dong Q, Qin L, Wu X, Zheng Y, Yang Y, et al: Identification of miRNomes in human liver and hepatocellular carcinoma reveals miR-199a/b-3p as therapeutic target for hepatocellular carcinoma. Cancer Cell 19: 232-243, 2011.

35. Bhaumik D, Scott GK, Schokrpur S, Patil CK, Campisi J and Benz CC: Expression of microRNA-146 suppresses NF-kappaB activity with reduction of metastatic potential in breast cancer cells. Oncogene 27: 5643-5647, 2008.

36. Halkein J, Tabruyn SP, Ricke-Hoch M, Haghikia A, Nguyen NQ, Scherr M, Castermans K, Malvaux L, Lambert V, Thiry M, et al: MicroRNA-146a is a therapeutic target and biomarker for peripartum cardiomyopathy. J Clin Invest 123: 2143-2154, 2013.

37. He H, Jazdzewski K, Li W, Liyanarachchi S, Nagy R, Volinia S, Calin GA, Liu CG, Franssila K, Suster S, et al: The role of microRNA genes in papillary thyroid carcinoma. Proc Natl Acad Sci USA 102: 19075-19080, 2005.

38. Shi G, Liu Y, Liu T, Yan W, Liu X, Wang Y, Shi J and Jia L: Upregulated miR-29b promotes neuronal cell death by inhibiting Bcl2L2 after ischemic brain injury. Exp Brain Res 216: 225-230, 2012.

39. Lu LF, Boldin MP, Chaudhry A, Lin LL, Taganov KD, Hanada T, Yoshimura A, Baltimore D and Rudensky AY: Function of miR-146a in controlling Treg cell-mediated regulation of Th1 responses. Cell 142: 914-929, 2010.

40. Cui Y, She K, Tian D, Zhang P and Xin X: miR-146a inhibits proliferation and enhances chemosensitivity in epithelial ovarian cancer via reduction of SOD2. Oncol Res 23: 275-282, 2016. 
41. Sapi E, Alvero AB, Chen W, O'Malley D, Hao XY, Dwipoyono B, Garg M, Kamsteeg M, Rutherford T and Mor G: Resistance of ovarian carcinoma cells to docetaxel is XIAP dependent and reversible by phenoxodiol. Oncol Res 14: 567-578, 2004.

42. Zhou P, Xu W, Peng X, Luo Z, Xing Q, Chen X, Hou C, Liang W, Zhou J, Wu X, et al: Large-scale screens of miRNA-mRNA interactions unveiled that the-3' UTR of a gene is targeted by multiple miRNAs. PLoS One 8: e68204, 2013.

43. Chen W, Zeng W, Li X, Xiong W, Zhang M, Huang Y, Zhou L and Jiang S: MicroRNA-509-3p increases the sensitivity of epithelial ovarian cancer cells to cisplatin-induced apoptosis. Pharmacogenomics 17: 187-197, 2016.

44. Chan WY, Cheung KK, Schorge JO, Huang LW, Welch WR, Bell DA, Berkowitz RS and Mok SC: Bcl-2 and p53 protein expression, apoptosis, and p53 mutation in human epithelial ovarian cancers. Am J Pathol 156: 409-417, 2000.

45. Qu Y, Xia P, Zhang S, Pan S and Zhao J: Silencing XIAP suppresses osteosarcoma cell growth, and enhances the sensitivity of osteosarcoma cells to doxorubicin and cisplatin. Oncol Rep 33: 1177-1184, 2015.

46. Chang JJ, Jeon SY, Song JY, Kim JH, Li L, Park DH, Lee YL, Park JJ, Woo DW, Kim GJ, et al: Alteration of X-linked inhibitors of apoptosis (XIAP) expression in rat model with DEN-induced hepatocellular carcinogenesis. Mol Cell Toxicol 4: 278-284, 2008.

47. Li X, Chen W, Zeng W, Wan C, Duan S and Jiang S: microRNA-137 promotes apoptosis in ovarian cancer cells via the regulation of XIAP. Br J Cancer 116: 66-76, 2017.

48. Tamm I, Kornblau SM, Segall H, Krajewski S, Welsh K, Kitada S, Scudiero DA, Tudor G, Qui YH, Monks A, et al: Expression and prognostic significance of IAP-family genes in human cancers and myeloid leukemias. Clin Cancer Res 6: 1796-1803, 2000.
49. Sasaki H, Sheng Y, Kotsuji F and Tsang BK: Down-regulation of $\mathrm{X}$-linked inhibitor of apoptosis protein induces apoptosis in chemoresistant human ovarian cancer cells. Cancer Res 60: 5659-5666, 2000.

50. Li J, Sasaki H, Sheng YL, Schneiderman D, Xiao CW, Kotsuji F and Tsang BK: Apoptosis and chemoresistance in human ovarian cancer: Is Xiap a determinant? Biol Signals Recept 9: 122-130, 2000.

51. Gyrd-Hansen M and Meier P: IAPs: From caspase inhibitors to modulators of NF-kappaB, inflammation and cancer. Nat Rev Cancer 10: 561-574, 2010.

52. Pang Y, Mao H, Shen L, Zhao Z, Liu R and Liu P: MiR-519d represses ovarian cancer cell proliferation and enhances cisplatin-mediated cytotoxicity in vitro by targeting XIAP. Onco Targets Ther 7: 587-597, 2014.

53. Chen W, Huang L, Hao C, Zeng W, Luo X, Li X, Zhou L, Jiang S, Chen $\mathrm{Z}$ and He Y: MicroRNA-155 promotes apoptosis in SKOV3, A2780, and primary cultured ovarian cancer cells. Tumour Biol 37: 9289-9299, 2016.

54. Liu S, Zhang P, Chen Z, Liu M, Li X and Tang H: MicroRNA-7 downregulates XIAP expression to suppress cell growth and promote apoptosis in cervical cancer cells. FEBS Lett 587: 2247-2253, 2013

55. Bell D, Berchuck A, Birrer M, Chien J, Cramer DW, Dao F, Dhir R, DiSaia P, Gabra H, Glenn P, et al; Cancer Genome Atlas Research Network: Integrated genomic analyses of ovarian carcinoma. Nature 474: 609-615, 2011. 